\title{
Transmisión de cargas entre forjados y puntales en un edificio de forjado reticular de casetón perdido utilizando clareado
}

\author{
Load transmission between slabs and shores in a building \\ with cast-in-place girderless hollow floor slab using \\ partial striking

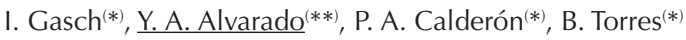

RESUMEN

En el presente artículo se presentan los resultados de la instrumentación llevada a cabo durante la construcción de un edificio de viviendas resuelto con forjados reticulares de casetón perdido situado en Sabadell (España), en el que se ha empleado un proceso de cimbrado, clareado, descimbrado (CCD). Se han registrado las cargas debidas a las operaciones constructivas de CCD en puntales de los seis primeros forjados. Dichos registros han permitido analizar la transmisión de cargas entre forjados y puntales durante la construcción de este edificio, teniendo un proceso de CCD. El análisis de las medidas experimentales ha permitido concluir que la transmisión de cargas entre forjados y puntales difiere según las condiciones de contorno del vano estudiado. Asimismo, se han comparado las medidas experimentales con diversos métodos simplificados que permiten simular la operación del clareado, obteniendo que el método que mejor se ajusta es el Nuevo Procedimiento Simplificado.

422-32

Palabras clave: Transmisión de cargas; clareado; estudio experimental; proceso constructivo; puntales; nuevo procedimiento simplificado.

\section{SUMMARY}

This paper presents the results of tests carried out during the construction of a building of flats with cast-in-place girderless hollow floor slab in Sabadell, Spain, using the shoring, clearing and striking (SCS) process. Loads on shores were recorded during the different construction stages of floor slabs 1 to 6 . The two first floor slabs had geometry different than the rest. The experimental results were used to analyse load transmission between slabs and shores during the construction of the building with a SCS process. The experimental results were compared with those obtained applying simplified methods that consider the real stiffness of the shoring, obtaining that the method with a better fit was the New Simplified Procedure.

Keywords: Load transmission; clearing; experimental study; construction process; shores; new simplified procedure.

\footnotetext{
(*) Universitat Politècnica de València (España)

(**) Pontificia Universidad Javeriana (Colombia)

Persona de contacto/Corresponding author: alvarado.y@javeriana.edu.co (Y. A. Alvarado)
} 


\section{INTRODUCCIÓN}

Cada año se construyen miles de metros cuadrados de estructura y forjados de edificios mediante cimbrado y descimbrado de plantas sucesivas, produciéndose el mayor número de colapsos durante el proceso constructivo (1) (2) (3). Buscando mejorar los plazos de construcción y los costes, sin reducir la seguridad estructural, en España se utiliza una técnica conocida como clareado, o descimbrado parcial. Esta técnica consiste en retirar el encofrado y la mitad de los puntales que soportan la losa unos días después de su hormigonado, pero sin que los puntales que permanecen pierdan en ningún momento su contacto con la estructura, reduciendo así el material necesario para el encofrado y el apuntalamiento.

Moragues et al. (4) (5) fueron los primeros en estudiar el efecto que tiene el clareado en la transmisión de cargas durante la construcción de forjados. Realizaron estudios tanto experimentales como numéricos, comparando los resultados obtenidos con los resultados del método simplificado de Grundy y Kabaila (6). Concluyeron que este método no es de aplicación para el caso particular del clareado.

Alvarado et al. (7) estudiaron de forma experimental el efecto del proceso de clareado. Para ello realizaron mediciones de cargas en puntales en un edificio experimental a escala real. A partir de estas mediciones observaron que una vez realizado el clareado, se produce una reducción de la carga media por unidad de área del sistema de apuntalamiento, la cual no es posible de evaluar con el método simplificado Grundy y Kabaila (6) al no considerar éste la rigidez real del sistema de apuntalamiento.

Posteriormente, Alvarado et al. (8) efectuaron una modelización por el método de los elementos finitos del edificio experimental. Dicho modelo tenía en cuenta el proceso constructivo seguido en el modelo experimental, así como la evolución de las propiedades del hormigón con el tiempo. La verificación del modelo se realizó a partir de los resultados obtenidos en el estudio experimental. Los investigadores concluyeron que la metodología adoptada en el desarrollo del modelo de elementos finitos era adecuada para representar la realidad.

Más recientemente, Calderón et al. (9) han desarrollado un nuevo procedimiento simplificado que permite estimar la transmisión de cargas entre forjados y puntales en los diferentes procesos constructivos.
Este nuevo procedimiento tiene en cuenta la rigidez real del conjunto de puntales y considera que la deformación media del conjunto de puntales bajo un forjado coincide con la deformación media de dicho forjado. Este nuevo procedimiento simplificado ha sido validado a partir de las medidas experimentales en puntales de Alvarado et al. (7) y a partir de los resultados de los modelos de elementos finitos desarrollados por Alvarado et al. (8). Los resultados obtenidos con el nuevo procedimiento simplificado se comparan también con otros procedimientos simplificados que también tienen en consideración la rigidez finita de los puntales, como son el método simplificado mejorado de Duan y Chen (10) y el método simplificado de Fang et al. (11) (12). Los investigadores concluyeron que el nuevo procedimiento simplificado presenta un mejor grado de ajuste con las medidas experimentales.

\section{OBJETIVOS Y NOVEDAD DEL ESTUDIO}

El presente estudio es continuación del trabajo previo Ilevado a cabo por el Instituto de Ciencia y Tecnología del Hormigón (ICITECH) de la Universitat Politècnica de València que se centra en el estudio de la transmisión de cargas entre forjados y puntales durante el proceso constructivo de edificios de hormigón armado (7) (9) (13).

En este artículo se presentan las mediciones llevadas a cabo durante la construcción de un edificio destinado a viviendas situado en Sabadell (España) con forjados reticulares de casetón perdido y donde se ha empleando un proceso de cimbrado, clareado y descimbrado (CCD). Estos resultados se comparan con los obtenidos al aplicar distintos procedimientos simplificados que permiten simular la operación de clareado, entre ellos el Nuevo Procedimiento Simplificado de Calderón et al. (9).

La principal novedad del presente trabajo es el estudio experimental de la transmisión de cargas entre forjados y puntales durante la construcción de un edificio de forjados reticulares de casetón perdido utilizando un proceso de cimbrado, clareado y descimbrado. Todos los estudios experimentales llevados a cabo hasta la fecha se han efectuado en edificios con forjados de losa maciza de hormigón armado (7) (13) o de forjado reticular con casetón recuperable (5). Además se han aplicado distintos métodos simplificados (9) (10) (11) para estimar la distribución de las cargas en los forjados y puntales originadas durante la construcción, comparando los resultados obtenidos con las medidas experimentales. 


\section{ESTUDIO EXPERIMENTAL}

\subsection{Descripción del edificio objeto de estudio}

El edificio donde se llevó a cabo el estudio está situado en Sabadell, Barcelona (España). Se trata de un edificio de 5 plantas y 2 sótanos, destinado a viviendas, donde la altura entre plantas varía entre $2,65 \mathrm{~m}$ y $4,45 \mathrm{~m}$.

Todas las plantas tienen forjado reticular de casetón perdido de $0,32 \mathrm{~m}$ de canto $(0,25+0,07)$, con un nervio de $0,15 \mathrm{~m}$ y casetón prefabricado de hormigón de $0,70 \mathrm{~m}$. Los balcones se resuelven con losa maciza de hormigón de 0,20 m de canto.

El hormigón utilizado para la construcción de todos los elementos del edificio tiene una resistencia característica a compresión de $25 \mathrm{MPa}$

\subsection{Proceso constructivo}

Los ciclos de construcción de la estructura quedaron definidos, para tres juegos de cimbras, con una planta cimbrada y dos clareadas.

El proceso de clareado consiste en la retirada de los tableros de encofrado, las sopandas secundarias y los puntales donde éstas se apoyan. En ese momento, el forjado queda apuntalado mediante las sopandas principales que transmiten la carga a los puntales que no han sido retirados.

En la Figura 1 se muestra el proceso de construcción seguido. Para cada forjado se diferencian tres fases: cimbrado, clareado, y descimbrado.

\subsection{Instrumentación}

Se instrumentó un único vano por planta con 2 sopandas principales. Dicho vano se corresponde con un vano de esquina en los forjados de sótano, donde además existe un muro perimetral paralelo a las sopandas, y con un vano extremo con voladizo en el resto de forjados (Figura 2). En total se instrumentaron 15 puntales en cada uno de los sótanos y 16 puntales por forjado en el resto de plantas (Figura 3). El vano instrumentado tenía unas dimensiones de 4,40 x 7,00 m, en los sótanos y de 4,60 x 7,00 m con un voladizo de 1,70 $\mathrm{m}$ en el resto de plantas.

Cada puntal estaba instrumentado con 3 bandas extensométricas dispuestas con un ángulo de $120^{\circ}$ y a una altura de $1,25 \mathrm{~m}$ de la base del puntal. El valor medio de la deformación de las tres bandas permitió establecer la carga a la que estaría sometido cada uno de los puntales durante la construcción de la estructura. Los puntales instrumentados se calibraron mediante ensayos a compresión centrada, estableciendo una relación entre las deformaciones y la carga transmitida al puntal. Las bandas extensométricas dispuestas sobre los puntales fueron protegidas frente a posibles golpes y frente a la humedad.

Se dispusieron dos puntales de referencia por planta con el objeto de medir el efecto de la temperatura ambiente sobre los puntales, las galgas extensométricas y el equipo de adquisición de datos. Sobre estos puntales no se impuso ningún tipo de coacción en sus extremos, por lo que no formaban parte del sistema de cimbrado del edificio.

Para la lectura de las medidas, se utilizó un módulo de adquisición de datos al cual se conectaban las bandas extensométricas, y un ordenador portátil equipado con un software desarrollado por personal de ICITECH.

Se realizaron mediciones discontinuas durante los procesos de hormigonado, clareado y descimbrado de cada uno de los forjados, desde el hormigonado del Forjado 1 (techo de sótano 2), hasta el hormigonado del Forjado 6 (techo de la tercera planta). Se midieron cargas hasta el hormigonado del Forjado 6 ya que en esta operación es donde se espera la mayor solicitación de cargas sobre los forjados inferiores donde se apoyan puntales.

El estudio experimental se inició el 4 de Diciembre de 2009 con el hormigonado del Forjado 1 y finalizó el 13 de Mayo de 2010 con el hormigonado del Forjado 6.

\subsection{Resumen y análisis de las mediciones}

En el estudio se tomaron medidas antes y después de cada operación constructiva (hormigonado, clareado y descimbrado) obteniendo así medidas instantáneas. Dichas medidas permiten conocer los incrementos de carga en puntales y forjados debido únicamente a las distintas operaciones constructivas. Se realizaron medidas desde el hormigonado del Forjado 1 hasta el hormigonado del Forjado 6.

En la Tabla 1 se muestran los resultados obtenidos en cada una de las fases de construcción. Para cada forjado, se muestra la carga media $\left(\mathrm{Q}_{\text {exp }}\right)$ sobre los puntales $\left(\mathrm{kN} / \mathrm{m}^{2}\right)$ y el coeficiente de carga en puntales $\left(\mathrm{C}_{\text {exp }}\right)$. La carga media $\left(Q_{\text {exp }}\right)$ se obtiene como la suma de la carga total de los puntales instrumentados dividida entre el área de influencia total 
1. Proceso constructivo

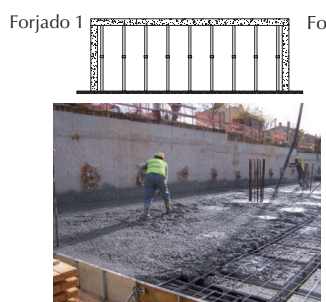

$\mathrm{t}=0$ días

Hormigonado F1

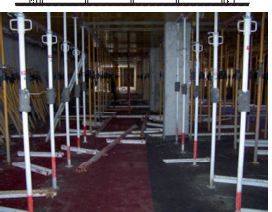

$\mathrm{t}=6$ días

Clareado F1

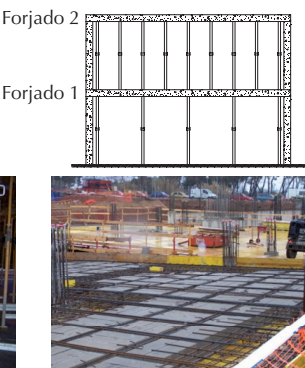

$t=53$ días

Hormigonado F2

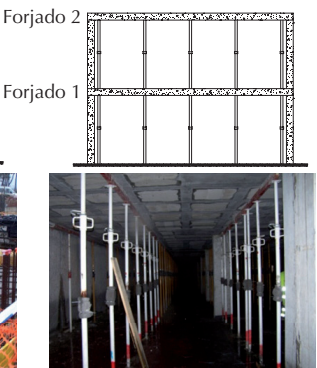

$\mathrm{t}=$ 59días

Clareado F2

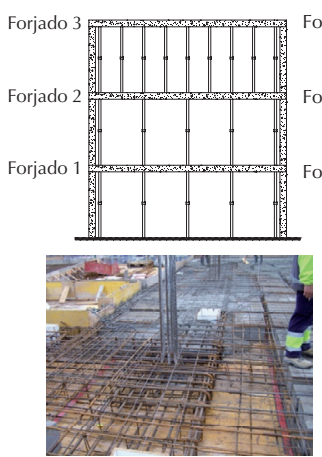

$\mathrm{t}=84$ días

Hormigonado F3

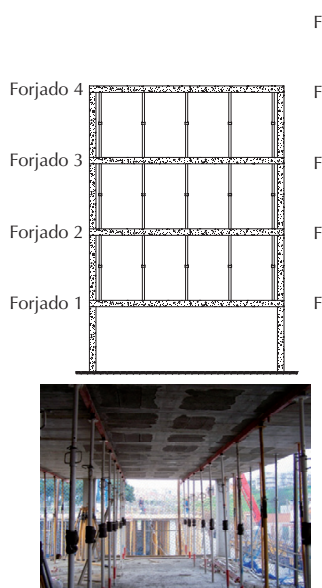

$\mathrm{t}=115$ días

Clareado F4

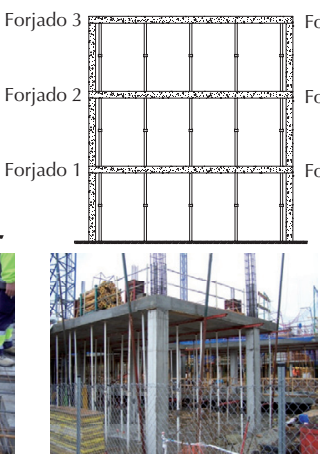

$\mathrm{t}=89$ días

Clareado F3

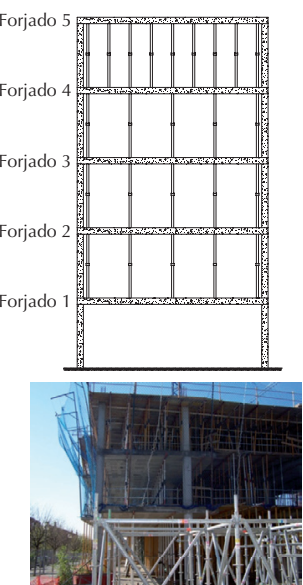

$\mathrm{t}=137$ días

Hormigonado F5

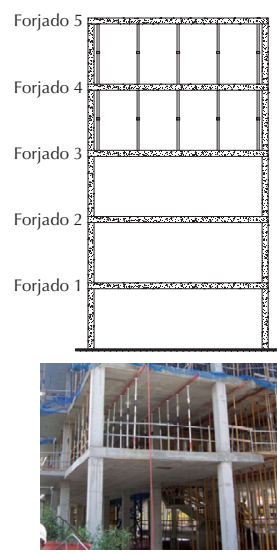

$\mathrm{t}=143$ días

Descimbrado F3

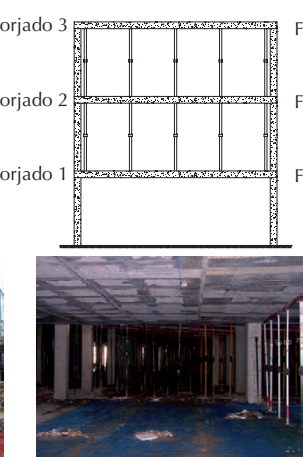

$\mathrm{t}=111$ días

Descimbrado F1
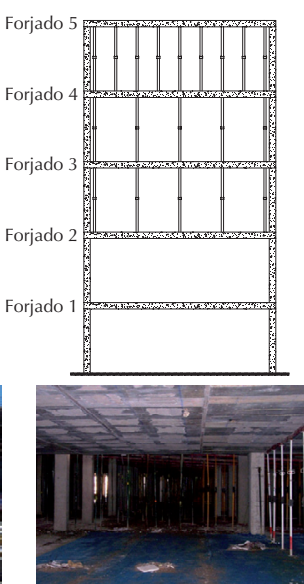

$\mathrm{t}=143$ días

Descimbrado F2

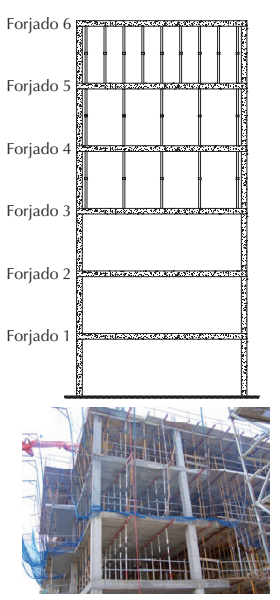

$\mathrm{t}=160$ días

Hormigonado F6

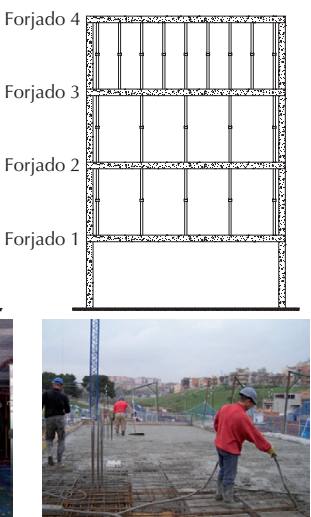

$\mathrm{t}=111$ días

Hormigonado F4

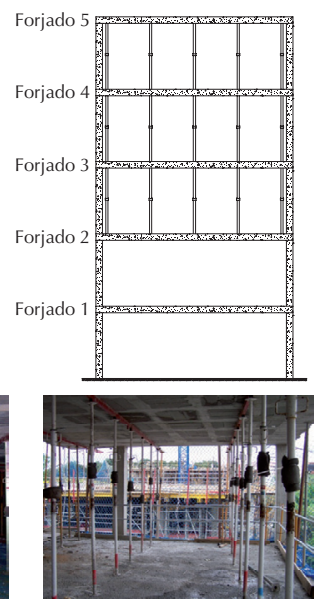

$\mathrm{t}=143$ días

Clareado F5 


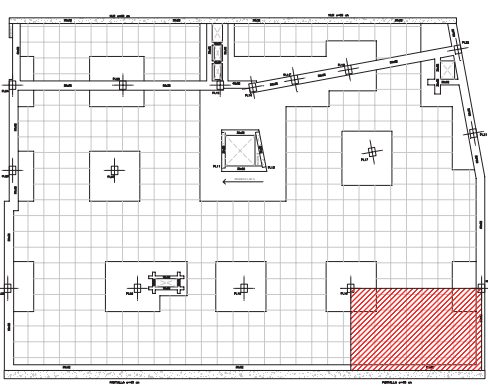

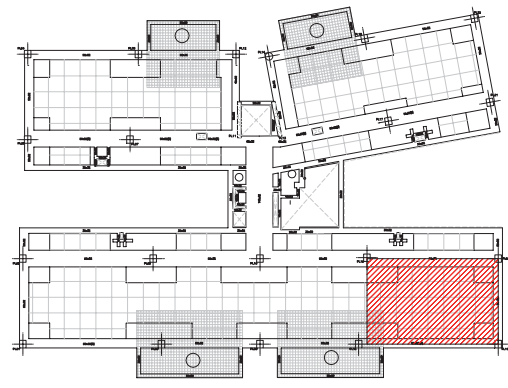

Forjados 3-6
2. Zonas instrumentadas.

3. Distribución de los puntales instrumentados.

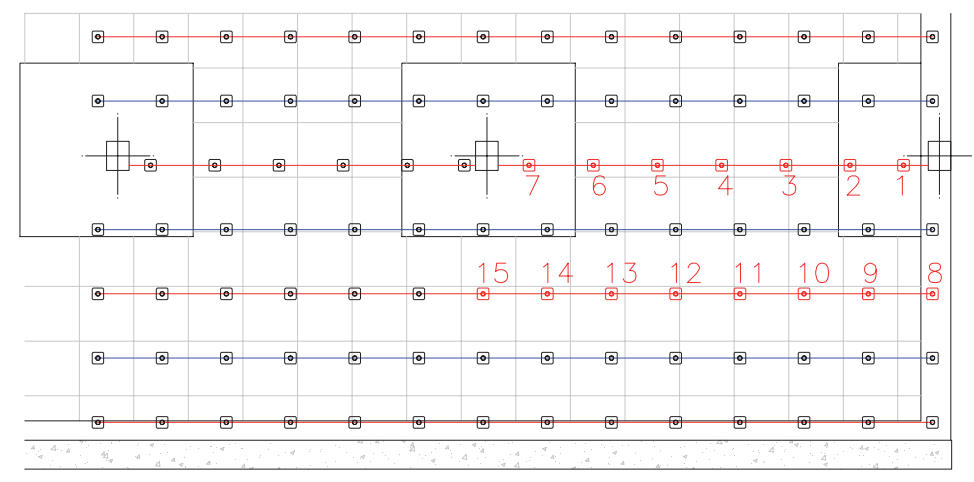

Forjados $1-2$

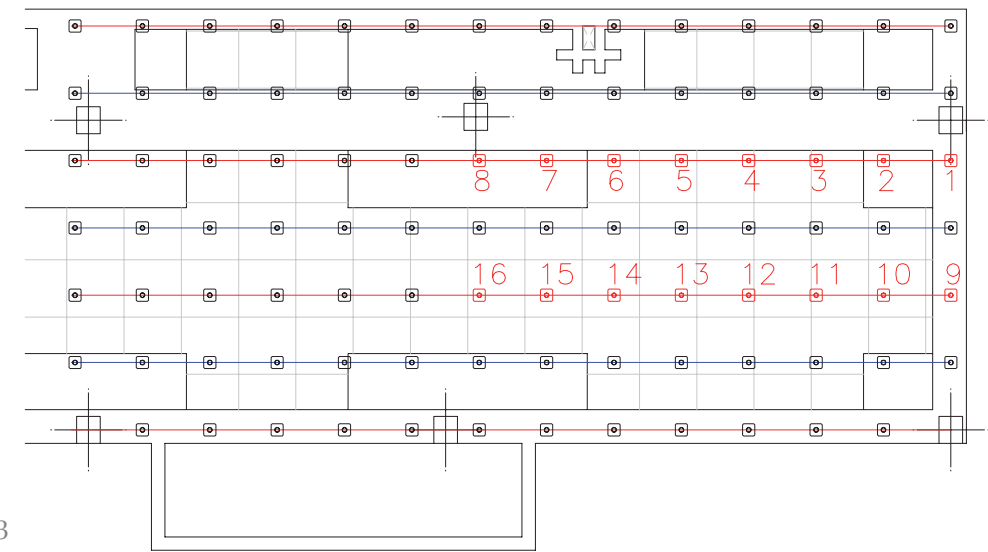

Forjados 3-6

de dichos puntales, mientras que el coeficiente de carga en puntales $\left(\mathrm{C}_{\text {exp }}\right)$ es la relación entre la carga media en puntales $\left(\mathrm{Q}_{\text {exp }}\right)$ y el peso propio del forjado considerando sólo el peso del hormigón $\left(3,39 \mathrm{kN} / \mathrm{m}^{2}\right)$.

De dichas mediciones experimentales se pueden extraer las siguientes conclusiones:

- Al realizar la operación de clareado, los forjados clareados asumen una fracción importante de carga.

- Vano de esquina (Forjados 1 y 2): $30-42 \%$ del peso propio del forjado.

- Vano extremo con voladizo (resto de forjados): $33-52 \%$ del peso propio del forjado.

- Al hormigonar un forjado superior sobre forjados inferiores ya clareados, una frac- ción importante de carga es asumida por el forjado sobre el que se apoyan los puntales del forjado hormigonado.

- Vano de esquina (Forjados 1 y 2): $69-73 \%$ del peso propio del forjado.

- Vano extremo con voladizo (resto de forjados): $47-69 \%$ del peso propio del forjado.

- Al retirar los puntales de un forjado o descimbrarlo, la carga que asumían dichos puntales se reparte entre los forjados superiores conectados por puntales. La carga asumida por el forjado inmediatamente superior al forjado descimbrado varió entre:

- Vano de esquina (Forjados 1 y 2): $57-67 \%$ de la carga que tenían los puntales retirados. 
Tabla 1. Resumen medición experimental

\begin{tabular}{|c|c|c|c|}
\hline Operación & Nivel & $\mathrm{Q}_{\exp }\left(\mathrm{kN} / \mathrm{m}^{2}\right)$ & $\mathrm{C}_{\text {exp }}$ \\
\hline Hormigonado Forjado 1 & 1 & 3,40 & 1,00 \\
\hline Clareado Forjado 1 & 1 & 1,97 & 0,58 \\
\hline Hormigonado Forjado 2 & $\begin{array}{l}2 \\
1\end{array}$ & $\begin{array}{l}3,40 \\
2,88\end{array}$ & $\begin{array}{l}1,00 \\
0,85\end{array}$ \\
\hline Clareado Forjado 2 & $\begin{array}{l}2 \\
1\end{array}$ & $\begin{array}{l}2,37 \\
2,27\end{array}$ & $\begin{array}{l}0,70 \\
0,67\end{array}$ \\
\hline Hormigonado Forjado 3 & $\begin{array}{l}3 \\
2 \\
1\end{array}$ & $\begin{array}{l}3,32 \\
3,36 \\
2,78\end{array}$ & $\begin{array}{l}0,98 \\
0,99 \\
0,82\end{array}$ \\
\hline Clareado Forjado 3 & $\begin{array}{l}3 \\
2 \\
1\end{array}$ & $\begin{array}{l}2,27 \\
2,37 \\
3,02\end{array}$ & $\begin{array}{l}0,67 \\
0,70 \\
0,89\end{array}$ \\
\hline Descimbrado Forjado 1 & $\begin{array}{l}3 \\
2\end{array}$ & $\begin{array}{l}1,53 \\
1,08\end{array}$ & $\begin{array}{l}0,45 \\
0,32\end{array}$ \\
\hline Hormigonado Forjado 4 & $\begin{array}{l}4 \\
3 \\
2\end{array}$ & $\begin{array}{l}3,39 \\
3,08 \\
1,73\end{array}$ & $\begin{array}{l}1,00 \\
0,91 \\
0,51\end{array}$ \\
\hline Clareado Forjado 4 & $\begin{array}{l}4 \\
3 \\
2 \\
\end{array}$ & $\begin{array}{l}1,62 \\
2,24 \\
1,39 \\
\end{array}$ & $\begin{array}{l}0,48 \\
0,66 \\
0,41 \\
\end{array}$ \\
\hline Hormigonado Forjado 5 & $\begin{array}{l}5 \\
4 \\
3 \\
2 \\
\end{array}$ & $\begin{array}{l}3,40 \\
3,41 \\
3,08 \\
1,97\end{array}$ & $\begin{array}{l}1,00 \\
1,01 \\
0,91 \\
0,58 \\
\end{array}$ \\
\hline Descimbrado Forjado 2 & $\begin{array}{l}5 \\
4 \\
3 \\
\end{array}$ & $\begin{array}{l}3,20 \\
3,27 \\
2,38 \\
\end{array}$ & $\begin{array}{l}0,94 \\
0,96 \\
0,78 \\
\end{array}$ \\
\hline Clareado Forjado 5 & $\begin{array}{l}5 \\
4 \\
3\end{array}$ & $\begin{array}{l}2,00 \\
2,64 \\
2,44\end{array}$ & $\begin{array}{l}0,59 \\
0,78 \\
0,72\end{array}$ \\
\hline Descimbrado Forjado 3 & $\begin{array}{l}5 \\
4\end{array}$ & $\begin{array}{l}1,91 \\
2,08\end{array}$ & $\begin{array}{l}0,56 \\
0,61\end{array}$ \\
\hline Hormigonado Forjado 6 & $\begin{array}{l}6 \\
5 \\
4\end{array}$ & $\begin{array}{l}3,40 \\
2,96 \\
2,09\end{array}$ & $\begin{array}{l}1,00 \\
0,87 \\
0,62\end{array}$ \\
\hline
\end{tabular}

- Vano extremo con voladizo (resto de forjados): $76 \%$ de la carga que tenían los puntales retirados.

- El reparto de cargas entre puntales no es uniforme. En general en los puntales de centro de vano se presentan los valores de carga máxima para cada una de las operaciones constructivas.

\section{APLICACIÓN DE DISTINTOS MÉTODOS SIMPLIFICADOS}

Para poder evaluar si los forjados son capaces de soportar con suficiente seguridad y sin deformaciones excesivas, los esfuerzos a los que va estar sometido durante el proceso de construcción, es necesario conocer la distribución de cargas en la estructura. Diversos son los métodos simplificados, propuestos por diferentes autores, para estimar dicha transmisión de cargas.

Para poder simular la operación del clareado, es necesario que el método simplificado considere la rigidez real de los puntales, ya que el clareado no es sino una reducción de dicha rigidez. Teniendo en cuenta esta condición, los métodos simplificados que se analizan son:

- Método Simplificado Mejorado (10).

- Método Simplificado de Fang et al. (11).

- Nuevo Procedimiento Simplificado (9).

Las cargas estimadas por dichos métodos son comparadas con las mediciones del estudio experimental.

\subsection{Resultados y comparación}

En la Tabla 2 se muestran se muestra una comparación de los coeficientes de carga en puntales determinados a partir del Método Simplificado Mejorado (10) $\left(C_{D \& C}\right)$, del Método Simplificado de Fang et al. (11) $\left(\mathrm{C}_{\text {Fang }}\right)$ y los determinados a partir del Nuevo Procedimiento Simplificado (9) $\left(\mathrm{C}_{\mathrm{NPS}}\right)$, con las medidas del estudio experimental $\left(\mathrm{C}_{\text {exp }}\right)$.

Como se muestra en la Tabla 2, el método que tiene un mejor ajuste con las mediadas experimentales es el Nuevo Procedimiento Simplificado de Calderón et al. (9), siendo la media del ratio $\mathrm{C}_{\text {exp }} / \mathrm{C}_{\mathrm{NPS}}$ para todas las fases de construcción es de 0,93 con una desviación típica de 0,15.

\section{CONCLUSIONES}

En este artículo se han detallado las medidas experimentales en puntales durante la construcción del edificio destinado a viviendas situado en Sabadell, España. Se trata de un edificio de forjado reticular de casetón perdido con muro de sótano. Las mediciones realizadas han permitido conocer cómo se transmitieron las cargas entre forjados y puntales durante la construcción del edificio en un proceso de cimbrado, clareado y descimbrado.

Las medidas experimentales han evidenciado que la transmisión de cargas entre forjados y puntales es diferente según el tipo vano analizado. Esto se debe a que las condiciones de contorno y geometría del vano hacen particular la deformabilidad y/o la rigidez de cada uno de los vanos del forjado. Independientemente del tipo vano, se observan unas pautas generales de comportamiento muy marcadas en cada una de las operaciones constructivas (hormigonado, clareado y descimbrado).

Las mediciones experimentales se han comparado con distintos métodos simplificados que permiten simular la operación de clareado, con el fin de validar un método simplificado que permita evaluar la transmisión de cargas durante un proceso de cimbrado, clareado y descimbrado. 
Tabla 2. Comparación entre los coeficientes de carga estimados con los distintos métodos simplificados y las medidas experimentales

\begin{tabular}{|c|c|c|c|c|c|c|c|}
\hline Operación & Nivel & $C_{D \& C}$ & $\mathrm{C}_{\text {exp }} / \mathrm{C}_{\mathrm{D \& C}}$ & $\mathrm{C}_{\text {Fang }}$ & $\mathrm{C}_{\text {exp }} / \mathrm{C}_{\text {Fang }}$ & $\mathrm{C}_{\mathrm{NPS}}$ & $\mathrm{C}_{\mathrm{exp}} / \mathrm{C}_{\mathrm{NPS}}$ \\
\hline Hormigonado Forjado 1 & 1 & 1,00 & 1,00 & 1,00 & 1,00 & 1,00 & 1,00 \\
\hline Clareado Forjado 1 & 1 & 0,38 & 1,53 & 0,24 & 2,37 & 0,64 & 0,90 \\
\hline Hormigonado Forjado 2 & $\begin{array}{l}2 \\
1 \\
\end{array}$ & $\begin{array}{l}1,00 \\
0,74 \\
\end{array}$ & $\begin{array}{l}1,00 \\
1,15\end{array}$ & $\begin{array}{l}1,00 \\
0,60 \\
\end{array}$ & $\begin{array}{l}1,00 \\
1,41\end{array}$ & $\begin{array}{l}1,00 \\
1,27 \\
\end{array}$ & $\begin{array}{l}1,00 \\
0,67\end{array}$ \\
\hline Clareado Forjado 2 & $\begin{array}{l}2 \\
1 \\
\end{array}$ & $\begin{array}{l}0,30 \\
0,49 \\
\end{array}$ & $\begin{array}{l}2,33 \\
1,38 \\
\end{array}$ & $\begin{array}{l}0,19 \\
0,31 \\
\end{array}$ & $\begin{array}{l}3,71 \\
2,15 \\
\end{array}$ & $\begin{array}{l}0,52 \\
0,97 \\
\end{array}$ & $\begin{array}{l}1,35 \\
0,69 \\
\end{array}$ \\
\hline Hormigonado Forjado 3 & $\begin{array}{l}3 \\
2 \\
1\end{array}$ & $\begin{array}{l}1,00 \\
0,58 \\
0,59\end{array}$ & $\begin{array}{l}0,98 \\
1,69 \\
1,39\end{array}$ & $\begin{array}{l}1,00 \\
0,54 \\
0,44\end{array}$ & $\begin{array}{l}0,98 \\
1,83 \\
1,87\end{array}$ & $\begin{array}{l}1,00 \\
1,02 \\
1,28\end{array}$ & $\begin{array}{l}0,98 \\
0,97 \\
0,64\end{array}$ \\
\hline Clareado Forjado 3 & $\begin{array}{l}3 \\
2 \\
1\end{array}$ & $\begin{array}{l}0,38 \\
0,41 \\
0,52\end{array}$ & $\begin{array}{l}1,78 \\
1,72 \\
1,70\end{array}$ & $\begin{array}{l}0,23 \\
0,27 \\
0,34\end{array}$ & $\begin{array}{l}2,88 \\
2,59 \\
2,61\end{array}$ & $\begin{array}{l}0,63 \\
0,83 \\
1,17\end{array}$ & $\begin{array}{l}1,06 \\
0,84 \\
0,76\end{array}$ \\
\hline Descimbrado Forjado 1 & $\begin{array}{l}3 \\
2 \\
\end{array}$ & $\begin{array}{l}0,33 \\
0,26 \\
\end{array}$ & $\begin{array}{l}1,37 \\
1,23 \\
\end{array}$ & $\begin{array}{l}0,20 \\
0,15 \\
\end{array}$ & $\begin{array}{l}2,31 \\
2,07 \\
\end{array}$ & $\begin{array}{l}0,44 \\
0,32 \\
\end{array}$ & $\begin{array}{l}1,01 \\
1,00\end{array}$ \\
\hline Hormigonado Forjado 4 & $\begin{array}{l}4 \\
3 \\
2\end{array}$ & $\begin{array}{l}1,00 \\
0,68 \\
0,35\end{array}$ & $\begin{array}{l}1,00 \\
1,34 \\
1,46\end{array}$ & $\begin{array}{l}1,00 \\
0,59 \\
0,26\end{array}$ & $\begin{array}{l}1,00 \\
1,55 \\
2,00\end{array}$ & $\begin{array}{l}1,00 \\
1,03 \\
0,54\end{array}$ & $\begin{array}{l}1,00 \\
0,89 \\
0,94\end{array}$ \\
\hline Clareado Forjado 4 & $\begin{array}{l}4 \\
3 \\
2\end{array}$ & $\begin{array}{l}0,42 \\
0,48 \\
0,30\end{array}$ & $\begin{array}{l}1,15 \\
1,39 \\
1,38\end{array}$ & $\begin{array}{l}0,27 \\
0,30 \\
0,18\end{array}$ & $\begin{array}{l}1,76 \\
2,19 \\
2,25\end{array}$ & $\begin{array}{l}0,63 \\
0,81 \\
0,46\end{array}$ & $\begin{array}{l}0,76 \\
0,81 \\
0,89\end{array}$ \\
\hline Hormigonado Forjado 5 & $\begin{array}{l}5 \\
4 \\
3 \\
2\end{array}$ & $\begin{array}{l}1,00 \\
0,81 \\
0,61 \\
0,33\end{array}$ & $\begin{array}{l}1,00 \\
1,25 \\
1,48 \\
1,74\end{array}$ & $\begin{array}{l}1,00 \\
0,74 \\
0,48 \\
0,23\end{array}$ & $\begin{array}{l}1,00 \\
1,37 \\
1,89 \\
2,54\end{array}$ & $\begin{array}{l}1,00 \\
1,24 \\
1,16 \\
0,59\end{array}$ & $\begin{array}{l}1,00 \\
0,82 \\
0,78 \\
0,98\end{array}$ \\
\hline Descimbrado Forjado 2 & $\begin{array}{l}5 \\
4 \\
3\end{array}$ & $\begin{array}{l}0,98 \\
0,76 \\
0,49\end{array}$ & $\begin{array}{l}0,96 \\
1,26 \\
1,59\end{array}$ & $\begin{array}{l}0,56 \\
0,50 \\
0,31\end{array}$ & $\begin{array}{l}1,67 \\
1,93 \\
2,53\end{array}$ & $\begin{array}{l}0,92 \\
1,06 \\
0,86\end{array}$ & $\begin{array}{l}1,02 \\
0,90 \\
0,91\end{array}$ \\
\hline Clareado Forjado 5 & $\begin{array}{l}5 \\
4 \\
3\end{array}$ & $\begin{array}{l}0,40 \\
0,54 \\
0,42\end{array}$ & $\begin{array}{l}1,48 \\
1,45 \\
1,71\end{array}$ & $\begin{array}{l}0,28 \\
0,36 \\
0,26\end{array}$ & $\begin{array}{l}2,12 \\
2,14 \\
2,76\end{array}$ & $\begin{array}{l}0,57 \\
0,86 \\
0,76\end{array}$ & $\begin{array}{l}1,04 \\
0,91 \\
0,94\end{array}$ \\
\hline Descimbrado Forjado 3 & $\begin{array}{l}5 \\
4 \\
\end{array}$ & $\begin{array}{l}0,19 \\
0,18 \\
\end{array}$ & $\begin{array}{l}2,93 \\
3,43 \\
\end{array}$ & $\begin{array}{l}0,24 \\
0,25 \\
\end{array}$ & $\begin{array}{l}2,35 \\
2,46 \\
\end{array}$ & $\begin{array}{l}0,44 \\
0,52 \\
\end{array}$ & $\begin{array}{l}1,28 \\
1,15\end{array}$ \\
\hline Hormigonado Forjado 6 & $\begin{array}{l}6 \\
5 \\
4\end{array}$ & $\begin{array}{l}1,00 \\
0,58 \\
0,31\end{array}$ & $\begin{array}{l}1,00 \\
1,49 \\
1,98\end{array}$ & $\begin{array}{l}1,00 \\
0,68 \\
0,40\end{array}$ & $\begin{array}{l}1,00 \\
1,27 \\
1,54\end{array}$ & $\begin{array}{l}1,00 \\
1,00 \\
0,77\end{array}$ & $\begin{array}{l}1,00 \\
0,87 \\
0,81\end{array}$ \\
\hline Media & - & & 1,51 & & 1,95 & & 0,93 \\
\hline Desviación Típica & - & & 0,52 & & 0,64 & & 0,15 \\
\hline
\end{tabular}

Se ha obtenido que el Nuevo Procedimiento Simplificado propuesto por Calderón et al (9) es el método que mejor grado de ajuste presenta con las medidas experimentales. Por lo tanto se puede considerar que este método es adecuado para estimar la transmisión de cargas entre forjados y puntales durante un proceso de cimbrado, clareado y descimbrado de edificios de forjados consecutivos de hormigón in situ similar al expuesto en el presente artículo.

\section{AGRADECIMIENTOS}

Los autores agradecen a las empresas ACSA y Encofrados J. Alsina por su inestimable cooperación para el desarrollo de esta investigación.

\section{REFERENCIAS}

(1) Kaminetzky, D.; Stivaros, P. (1994). Early-age concrete: construction loads, behavior, and failures. Concrete International, 16(1): 58-63.

(2) Kaminetzky, D. (1991). Design and construction failures: Lessons from forensic investigations. MacGraw-Hill, New York.

(3) Epaarachchi, D.; Stewart, M.; Rosowsky, D. (2002). Structural reliability of multistory buildings during construction. Journal of Structural Engineering, 128(2): 205-213, doi: 10.1061/ (ASCE)0733-9445(2002)128:2(205).

(4) Moragues, J. J.; Catalá, J.; Pellicer, E. (1996). An analysis of concrete framed structures during the construction process. Concrete International, 18(11): 44-48. 
(5) Moragues, J. J.; Catalá, J.; Salort, V.; Sirvent, P. L. (1991). Transmisión de cargas entre forjados, durante el proceso constructivo: Medidas realizadas en obra. Hormigón y Acero, 179: 83-91.

(6) Grundy, P.; Kabaila, A. (1963). Construction loads on slabs with shored formwork in multistory buildings. ACl Journal Proceedings, 60(12): 1729-1738.

(7) Alvarado, Y. A.; Calderón, P. A.; Adam, J. M.; Payá, I. J.; Pellicer, T.; Pallares, F. J.; Moragues, J. J. (2009). An experimental study into the evolution of loads on shores and slabs during construction of multistory buildings using partial striking. Engineering Structures, 31(9): 2132-2140, doi:10.1016/j.engstruct.2009.03.021.

(8) Alvarado, Y. A.; Calderón, P. A.; Gasch, I.; Adam, J. M. (2010). A numerical study into the evolution of loads on shores and slabs during construction of multistorey buildings. Comparison of partial striking with other techniques. Engineering Structures, 32(10): 3093-3102, doi:10.1016/j.engstruct.2010.05.028.

(9) Calderón, P. A.; Alvarado, Y. A.; Adam, J. M. (2011). A new simplified procedure to estimate loads on slabs and shoring during the construction of multistorey buildings. Engineering Structures, 33(5): 1565-1575, doi: 10.1016/j.engstruct.2011.01.027.

(10) Duan, M. Z.; Chen, W. F. (1995). Improved simplified method for slab and shore load analysis during construction. Project report CE-STR-95-24, Purdue University, West Lafayette, Ind.

(11) Fang, D. P.; Zhu, H. Y.; Geng, C. D.; Liu, X. L. (2001). Floor load distribution in reinforced concrete buildings during construction. ACI Structural Journal, 98(2): 149-156.

(12) Fang, D. P.; Zhu, H. Y.; Geng, C. D.; Liu, X. L. (2001). On-site measurments of structural characteristics of reinforced concrete buildings during construction. ACI Structural Journal, 98(2): 157-163.

(13) Alvarado, Y.A. (2009). Estudio experimental y numérico de la construcción de forjados hormigonados in situ mediante procesos de cimbrado, clareado y descimbrado de plantas consecutivas (Tesis doctoral). Valencia: Universidad Politécnica de Valencia. Disponible en http://hdl.handle.net/10251/7285. 